\title{
Reconhecimento de Dígitos Manuscritos em Vídeos Educacionais Utilizando Florestas Aleatórias
}

\author{
Paulo R. S. Borges, Ismar F. Silveira, Leandro Nunes de Castro \\ Programa de Pós-Graduação em Engenharia Elétrica e Computação (PPGEEC) \\ Universidade Presbiteriana Mackenzie - São Paulo, SP - Brasil \\ paulorenato.borges@mackenzista.com.br, \\ ismar.silveira@mackenzie.br, Inunes@mackenzie.br
}

\begin{abstract}
This paper aims to present a study on the working of random forests and verify their accuracy for handwritten digits classification contained in images. A modular architecture was built to train a random forest, preprocess an image and predict the information contained in that image. Furthermore, experiments were performed with the architecture implemented by adjusting the input parameters to analyze the random forest accuracy. The obtained results provided satisfying accuracy and validated the random forest, which can aid in the automatic semantic annotation of educational videos.
\end{abstract}

Resumo. Este trabalho tem como objetivo apresentar um estudo sobre o funcionamento das florestas aleatórias e verificar sua acurácia para a classificação de dígitos escritos à mão contidos em imagens. Foi construída uma arquitetura com módulos para treinamento de uma floresta aleatória, préprocessamento de uma imagem e predição das informações contidas nessa imagem. Foram realizados testes com a implementação dessa arquitetura ajustando parâmetros de entrada com o intuito de analisar a acurácia da floresta aleatória. Os valores obtidos, além de fornecerem acurácia satisfatória da floresta aleatória, validaram essa arquitetura que pode auxiliar na anotação semântica automática de vídeos educacionais.

\section{Introdução}

Aprendizado de Máquina (AM) é a área dedicada ao desenvolvimento e aplicação de processos computacionais automáticos que permitem que um computador aprenda a realizar uma determinada tarefa. Desse modo, ela fornece subsídios potenciais para a construção de sistemas de alto desempenho e com aplicações diversas. Diferentes tipos de algoritmos destinados ao aprendizado de máquina foram desenvolvidos, muitas vezes com foco em sua capacidade de generalização. Dentre esses algoritmos encontram-se os que utilizam como modelo árvores de decisão (Mitchell, 1997; de Castro; Ferrari, 2016). As árvores de decisão são algoritmos preditivos que implementam um mecanismo de tomada de decisão em forma de árvore, mas que podem ser traduzidos em regras de decisão. Sua aplicação pode ser em problemas de classificação ou estimação.

Os algoritmos de indução de árvores de decisão precisam do tratamento de questões práticas como: determinar quando parar o crescimento da árvore, escolher uma medida de seleção de atributos apropriada, manipular atributos contínuos, manusear dados de treinamento com ausência de valores de atributo, manipular atributos com diferentes custos, tratar o sobreajuste das regras e melhorar a eficiência computacional 
(Mitchell, 1997). O sobreajuste ocorre quando o modelo se ajusta aos desvios causados por erros de medição ou fatores aleatórios (ruídos) nos dados. Formalmente, dado um espaço de hipóteses $H=\left\{\boldsymbol{h}_{1}, \boldsymbol{h}_{2}, \ldots \boldsymbol{h}_{N}\right\}$, isto é, $\mathrm{H}=\left\{\boldsymbol{h}_{i}\right\}_{i=1}^{N}$, um modelo de predição sobreajustado $M_{s}$ possui uma hipótese $\boldsymbol{h}_{i}$ que tem erro $\boldsymbol{e}$ menor que o erro $\boldsymbol{e}^{\prime}$ de $\boldsymbol{h}_{i}^{\prime}$ para as amostras de treinamento, mas $\boldsymbol{h}_{i}^{\prime}$ tem erro $\boldsymbol{e}^{\prime}$ menor que $\boldsymbol{e}$ de $\boldsymbol{h}_{i}$ sobre toda a distribuição do conjunto de dados, ou seja, $\boldsymbol{e}\left(\boldsymbol{h}_{i}\right)<\boldsymbol{e}^{\prime}\left(\boldsymbol{h}_{i}^{\prime}\right)$ para os dados de treinamento, mas $\boldsymbol{e}^{\prime}\left(\boldsymbol{h}_{i}^{\prime}\right)<\boldsymbol{e}\left(\boldsymbol{h}_{i}\right)$ para toda a distribuição dos dados (Mitchell, 1997).

Uma das causas do sobreajuste é a proporção do número de características em relação ao número de amostras de treinamento, ou seja, se uma árvore de decisão é treinada com poucas amostras, mas tem espaço dimensional elevado, é alta a probabilidade de ocorrer sobreajuste (Mitchell, 1997; Patti et al, 2015). Nesse contexto, foram desenvolvidas diversas técnicas utilizando critérios de divisão e otimização do tamanho da árvore para tratar problemas de sobreajuste em algoritmos baseados em árvores de decisão. Entretanto, muitas delas acarretam perda da acurácia desses algoritmos para o conjunto de testes (Ho, 1995). Estudos como o de Ho (1995); Ho (1998) e Amit et al. (2000) foram realizados para o tratamento de sobreajuste utilizando múltiplas árvores e serviram de subsídio para a idealização, por Breiman (2001), do método denominado florestas aleatórias de decisão, tema do presente estudo.

Dentre os problemas de aprendizagem de máquina em que as florestas aleatórias de decisão podem ser empregadas, evidencia-se o de aprender a partir de imagens e classificá-las (Ristin et al, 2016; Pu et al, 2015). Nesse cenário, as florestas aleatórias de decisão podem ser utilizadas para a classificação de informações e características extraídas de imagens e, mais especificamente, para o reconhecimento de caracteres escritos à mão, contidos em imagens (Simon, Adam e Heutte, 2007; Ho, 1995; Yao et al, 2014). O reconhecimento de caracteres manuscritos envolve diversos procedimentos que vão desde o processamento da imagem para extração de características, passando pelo reconhecimento de caracteres individuais, até a análise sintática e semântica com o objetivo de verificar o contexto no qual o texto escrito se insere.

Borges (2015) aponta que em variados segmentos, inclusive na educação, estão sendo utilizadas ferramentas semânticas. Assim, os objetos de aprendizagem, como vídeos educacionais, por exemplo, se construídos com anotação semântica adequada podem ser guardados em repositórios de modo a serem reutilizados e referenciados no meio educacional. Entretanto, de acordo com Borges (2015), o processo de anotação feito manualmente é trabalhoso e demanda tempo. Nesse sentido, as florestas aleatórias podem ser utilizadas para tarefas de classificação e processamento de imagens contidas em vídeos para a extração de informações, auxiliando assim no registro e na recuperação de conteúdos audiovisuais em um portal semântico educacional. Nessa direção, este trabalho tem como objetivo apresentar um estudo sobre o funcionamento das florestas aleatórias e verificar sua acurácia para a classificação de dígitos escritos à mão contidos em imagens.

Esse artigo está organizado da seguinte forma. A Seção 2 faz uma breve introdução sobre florestas aleatórias e a Seção 3 apresenta a aplicação de florestas aleatórias no reconhecimento de caracteres manuscritos em imagens. A descrição do experimento está apresentada na Seção 4 e a análise de resultados é mostrada na Seção 5. As considerações finais deste estudo estão na Seção 6 com uma discussão sobre os resultados obtidos e a perspectiva de trabalhos futuros. 
V Congresso Brasileiro de Informática na Educação (CBIE 2016)

Anais dos Workshops do V Congresso Brasileiro de Informática na Educação (CBIE 2016)

\section{Florestas Aleatórias de Decisão}

A utilização de múltiplas árvores de decisão foi proposta por Ho (1995), que estabeleceu divisões das árvores com hiperplanos oblíquos para essas florestas de árvores. Posteriormente, Ho (1998) apontou que outros métodos de divisão se comportam de modo similar, desde que sejam forçados aleatoriamente para apresentarem insensibilidade a algumas dimensões. Observou-se que uma floresta com número de árvores maior pode melhorar a acurácia para o conjunto de teste. Dietterich (1998) lançou a ideia da seleção aleatória de características para a divisão de um nó escolhida aleatoriamente dentre as $K$ melhores divisões. O que se percebe de elemento comum desses trabalhos é que para a $k$ ésima árvore, um vetor aleatório $\Theta_{k}$ é gerado a partir dos dados do conjunto de treinamento $C$, tornando-se um subconjunto de $C$, independentemente dos vetores $\Theta_{1}, \ldots, \Theta_{k-1}$ gerados anteriormente, mas com a mesma distribuição. Esses trabalhos vieram influenciar na idealização das florestas aleatórias propostas por Breiman (2001).

Uma floresta aleatória é um classificador constituído de uma coleção de classificadores estruturados em árvores $\left\{h\left(x, \Theta_{\mathrm{k}}\right), k=1, \ldots\right\}$, onde os $\left\{\Theta_{\mathrm{k}}\right\}$ são vetores aleatórios identicamente distribuídos amostrados a partir da base de dados e cada árvore fornece um voto unitário para a classe mais popular de uma entrada $z_{i}=\left(x_{i}, y_{i}\right)$. Dado um conjunto de treinamento $C$ composto por amostras $z$, que possuem um conjunto de características $M$, uma floresta aleatória $\mathrm{F}$ pode ser formada de acordo com o algoritmo apresentado no Quadro 1, a seguir (Breiman, 2001).

\section{Quadro 1. Algoritmo de Construção de uma Floresta Aleatória.}

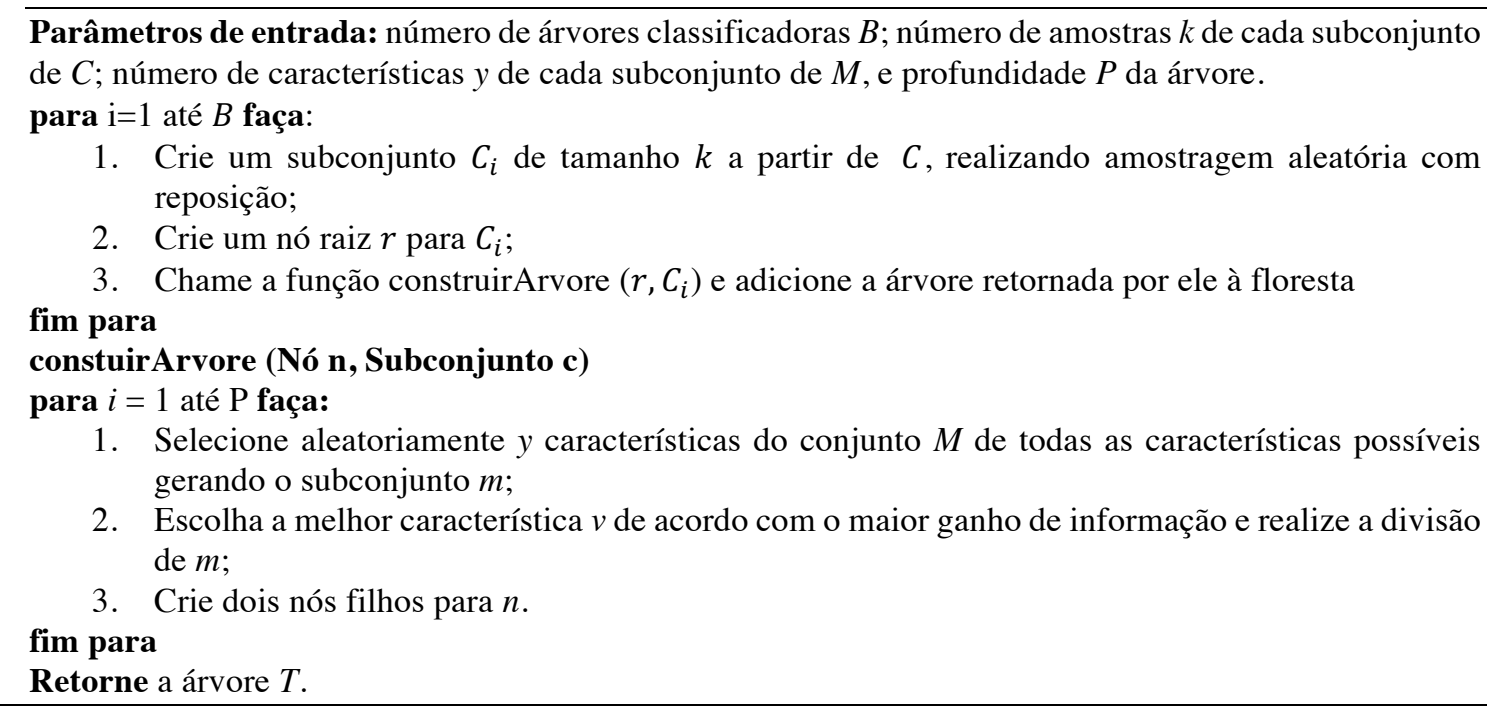

Depois de construir a floresta aleatória, para realizar a predição de um novo dado de entrada é necessário efetuar o somatório dos votos individuais de cada árvore $T$ pertencente ao conjunto de árvores que forma a floresta aleatória $\mathrm{F}$.

\subsection{Principais Características das Florestas Aleatórias}

Florestas aleatórias são formadas por árvores que crescem a partir de entradas ou combinações de entradas selecionadas aleatoriamente. Isso é possibilitado pela utilização da metodologia bagging (Bootstrap Aggregating) para gerar os subconjuntos de treinamento. Dado um conjunto de características $M$ de tamanho $t$, o bagging produz subconjuntos de características $M_{i}$, cada um com tamanho $t$ ', amostrando $M$ 
uniformemente e com reposição, o que significa que uma característica $m \in M$ pode ser uma amostra em mais de um $M_{i}$. A utilização de bagging aumenta a acurácia da floresta aleatória e possibilita obter estimativas internas do erro de generalização da floresta, da força de uma árvore classificadora e correlação entre as árvores classificadoras, utilizando o método out-of-bag. Dado um conjunto de treinamento $C$, para cada objeto $c_{i}=\left(x_{i}, y_{i}\right)$ $\in C$, uma árvore classificadora $T$ da floresta aleatória utilizando $c_{i}$ é construída efetuando as médias dos votos apenas das árvores classificadoras que não correspondem ao bootstrap de amostras que contenham $c_{i}$. Esse processo é denominado out-of-bag e fornece estimativas de erro que dispensam a utilização de um conjunto de teste, sendo equivalente à validação cruzada, mas calcula as estimativas internas durante $o$ treinamento (Breiman, 2001).

A importância de uma variável e a correlação entre classificadores também são estimadas usando out-of-bag e são utilizadas para medir a força de predição dessa variável. Suponha que há $t$ variáveis de entrada. Depois que cada árvore é construída, os valores das variáveis $m_{i}$ das amostras out-of-bag são aleatoriamente permutados e essas amostras out-of-bag são rodadas na árvore correspondente. A classificação dada para cada variável $m_{i}$ que é out-of-bag é salva. Isso é repetido para $m=1,2, \ldots, t$. Ao final da execução, a pluralidade dos votos de classe para $m_{i}$ é comparada com o número de votos para a classe correta dos dados out-of-bag para então calcular a taxa de classificação incorreta. A saída é a percentagem de aumento na taxa de classificação incorreta, em comparação com a taxa obtida com as amostras out-of-bag (Breiman, 2001). A escolha da melhor variável efetuada no algoritmo de construção de uma árvore da floresta aleatória é realizada conforme o valor calculado para sua importância na floresta (Friedman, Hastie, Tibshirani, 2001).

As florestas aleatórias podem ser utilizadas tanto para a classificação quanto para a regressão e não são sensíveis a ruídos como árvores individuais, já que são formadas por árvores que foram construídas a partir de amostras com reposição para a diminuição da correlação entre as árvores (Breiman, 2001). Essa correlação também pode ser ajustada de acordo com o número escolhido para $m$, ou seja, quanto menor o valor de $m$, menor a correlação entre qualquer par de árvores que compõem a floresta e, consequentemente, menor a variância da média das predições tanto para classificação quanto para regressão. A robustez das florestas aleatórias em relação a ruídos, outliers e ao problema de sobreajuste é alcançada pela utilização de metodologias que diminuem a variância do modelo utilizado (Friedman, Hastie, Tibshirani, 2001).

\section{Florestas Aleatórias Aplicadas ao Reconhecimento de Dígitos Manuscritos}

Para sistemas de visão computacional, a tarefa de extração de informações contidas em imagens e classificação dessas informações é complexa. Assim, como o propósito deste estudo é verificar a acurácia de florestas aleatórias aplicadas ao reconhecimento de caracteres manuscritos, foi limitado o escopo de processamento de vídeos para imagens que possuam dígitos numéricos encontrados em vídeos educacionais. Para tanto, foi construída uma arquitetura para o treinamento da floresta aleatória, extração de informações de uma imagem e predição do conteúdo dessa imagem pela floresta aleatória. 
V Congresso Brasileiro de Informática na Educação (CBIE 2016)

Anais dos Workshops do V Congresso Brasileiro de Informática na Educação (CBIE 2016)

\subsection{Algoritmo para o treinamento e classificação com florestas aleatórias}

A arquitetura construída para o treinamento e classificação com florestas aleatórias é composta por três módulos: módulo de treinamento; módulo de pré-processamento; e módulo de classificação (Figura 1). Esses módulos foram implementados utilizando OpenCV 3.1 e $C++$.

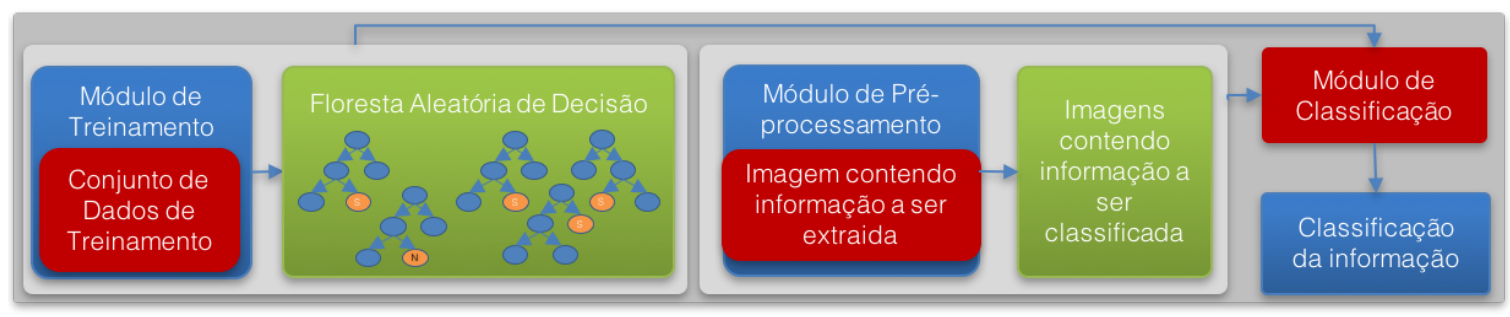

Figura 1. Arquitetura do sistema

Para o treinamento da floresta aleatória elegeu-se o banco de dados MNIST, contendo 60.000 imagens, destinado à classificação de dígitos numéricos. Cada imagem possui um dígito escrito em branco com fundo preto e tamanho $28 \times 28$ pixels (Figura 2).

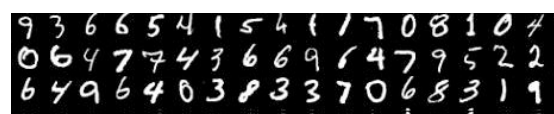

Figura 2. Amostra de imagens contidas no conjunto de treinamento

Assim, empregando essa base de dados, foi construído o módulo de treinamento, conforme o Algoritmo apresentado no Quadro 2 a seguir. Para gerar a floresta aleatória, foi utilizada a classe RTrees (OpenCV), a qual foi fundamentada no algoritmo anterior

\section{Quadro 2: Algoritmo do Módulo de treinamento}

\footnotetext{
Parâmetros de entrada: base de dados contendo as imagens de treinamento

Varrer recursivamente a base de dados e encontrar todas as imagens de treinamento I contidas nela. para cada imagem $\mathbf{i} \in \mathbf{I}$ faça:

1. Converter a matriz contendo os dados de $\mathbf{i}$ para um vetor $\mathbf{v}$;

fim para.

2. Adicionar $\mathbf{v}$ à matriz de dados de treinamento $C$;

Gerar a floresta aleatória passando como parâmetro o conjunto de treinamento $C$, a profundidade máxima da árvore $P$, o tamanho do subconjunto de treinamento $k$, o número de variáveis $y$ de cada subconjunto de todas as possíveis categorias, e o número de árvores classificadoras $B$.

Salve o arquivo contendo as informações da floresta aleatória: número de árvores; profundidade máxima da árvore; tamanho dos subconjuntos utilizados para treinamento; número de variáveis utilizadas para amostragem do conjunto de características; importância de cada variável utilizada para o treinamento; taxa de erro de classificação; classes resultantes do treinamento e estrutura da floresta aleatória.

fim Algoritmo.
}

Como dado de saída desse módulo, foi gerado um arquivo $\mathrm{xml}$ contendo as informações sobre a floresta aleatória.

Após a construção da floresta aleatória, foi implementado o módulo de préprocessamento para preparar uma imagem nos padrões das imagens utilizadas para treinamento e, assim, tornar possível submeter a imagem para predição por essa floresta aleatória. Como o foco deste estudo é a verificação da acurácia das florestas aleatórias, foi estabelecido que as imagens de entrada para esse módulo teriam fundo preto e informações em branco, conforme Figura 3. 


\title{
0,4
}

Figura 3. Exemplo de entrada do módulo de pré-processamento

Ao receber uma imagem, contendo um ou mais dígitos, esse módulo de préprocessamento funciona de acordo com o algoritmo de pré-processamento, apresentado no Quadro 3.

\section{Quadro 3: Algoritmo de Pré-processamento de uma imagem}

\author{
Requerido: imagem a ser processada \\ Transformar a imagem de RGB para escala de cinza; \\ Encontrar os contornos $E$ das informações da imagem; \\ para cada contorno $e \in E$ faça: \\ 1. Dilatar o contorno com o intuito de aproximá-lo a um retângulo; \\ 2. Copiar as informações da imagem contidas na área do retângulo; \\ 3. Redimensionar essas informações para uma matriz de $28 \times 28$ pixels. \\ 4. Salvar essas informações em uma nova imagem. \\ fim para. \\ fim Algoritmo.
}

Inicialmente, é realizada a transformação do padrão RGB para a escala de cinza, com o intuito de tornar o problema mais simples e melhorar os resultados nas fases seguintes. Prosseguindo, são identificados os contornos de cada informação em destaque na imagem e cada contorno é salvo em uma nova imagem, de acordo com o requerido pela floresta aleatória para a classificação, conforme Figura 4.

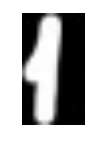

Figura 4. Exemplo de saída do módulo de pré-processamento

Na sequência, o módulo de classificação recebe uma imagem com características semelhantes ao conjunto de treinamento e funciona de acordo com o algoritmo de Predição, apresentado no Quadro 4.

\section{Algoritmo 4: Algoritmo para Predição}

Parâmetros de entrada: caminho do arquivo contendo as informações da floresta aleatória; caminho para a imagem a ser classificada

Ler a imagem de entrada e salvar o resultado em uma matriz bidimensional $i$;

Transformar $i$ em um vetor $v$;

Realizar a predição da informação contida em $v$ passando como parâmetro $v$ e o caminho do arquivo contendo as informações da floresta aleatória.

Imprimir o resultado da classificação realizada pela floresta aleatória para a informação de $v$.

fim Algoritmo.

Como saída do módulo de classificação é fornecida a predição da classificação da informação contida na imagem de entrada.

Na sequência da implementação do algoritmo para o treinamento e classificação de dígitos utilizando florestas aleatórias partiu-se para os experimentos.

\section{Experimentos}

Com o intuito de verificar a adequação da implementação da arquitetura proposta para a utilização de florestas aleatórias no reconhecimento de dígitos manuscritos, foram 
V Congresso Brasileiro de Informática na Educação (CBIE 2016)

Anais dos Workshops do V Congresso Brasileiro de Informática na Educação (CBIE 2016)

realizados testes preliminares e, em seguida, foram efetuados os experimentos com foco na acurácia da floresta aleatória.

\subsection{Validação da Implementação do Algoritmo para o Treinamento e Classificação}

Para os testes preliminares foram geradas 20 imagens com resoluções variadas contendo um ou mais dígitos seguindo os requisitos do módulo de pré-processamento. Além disso, foram treinadas e salvas 10 florestas aleatórias utilizando o banco de dados MNIST. Essas 20 imagens foram submetidas ao módulo de pré-processamento, que criou as imagens redimensionadas dos dígitos individuais, de acordo com o requerido pelo módulo de predição. Assim, foi invocado o módulo de predição passando como parâmetro o diretório contendo as imagens dos dígitos individuais e o caminho para o arquivo contendo as informações da floresta aleatória. Essa chamada foi realizada 10 vezes, uma para cada floresta aleatória gerada anteriormente.

A análise dos resultados desses testes possibilitou verificar a adequação do algoritmo implementado, bem como a identificação de valores para os parâmetros de entrada para o treinamento da floresta aleatória que apresentaram acurácia maior que 92\%. Esses testes forneceram subsídios para estabelecer informações consideradas nos experimentos descritos a seguir.

\subsection{Experimentos com o Ajuste dos Parâmetros da Floresta Aleatória}

Como experimento, este estudo buscou ajustar dois parâmetros das florestas aleatórias: o número $B$ de árvores da floresta; e o número de amostras $y$ de cada subconjunto de características de $M$, objetivando encontrar um valor desses parâmetros adequado para uma acurácia satisfatória da taxa de reconhecimento de caracteres numéricos em imagens utilizando o sistema proposto.

\subsubsection{Instrumentos}

Para a realização dos experimentos foi utilizada a implementação em $O p e n C V$ e $C++\operatorname{dos}$ módulos de treinamento, pré-processamento e classificação, rodando em um MacBook Pro, com processador Intel Core i7 (2.3 GHz, $8 \mathrm{~GB}$ de RAM), com OS X El Captain. Como base de dados para o treinamento foram utilizadas 7792 imagens (Referidas como conjunto de treinamento $C$ ) no formato .png e, para a realização dos testes de predição, 2000 imagens (Referidas como conjunto de teste $T$ ) no mesmo formato.

\subsubsection{Procedimentos}

Testes de variação do número de árvores de uma floresta aleatória foram realizados por Ho (1995), utilizando árvores construídas com algoritmos baseados em divisões de nós utilizando hiperplanos. Bernard, Adam e Heutte (2007) atribuem diferentes valores para $y$ em experimentos que visam um aumento progressivo desse valor, afirmando que esse valor não tem que aumentar muito. Além disso, para Breiman (2001), na seleção aleatória da divisão existe uma série de números inteiros aleatórios independentes localizados no intervalo $[1, y]$.

Fundamentando nesses conceitos, o experimento foi iniciado estabelecendo dois intervalos de valores de $y$ e $B$. Foram selecionados seis valores de 5 a 250 para $B$, os quais foram escolhidos conforme a tendência empírica da porcentagem de acurácia que emergiu 
V Congresso Brasileiro de Informática na Educação (CBIE 2016)

Anais dos Workshops do V Congresso Brasileiro de Informática na Educação (CBIE 2016)

no decorrer dos experimentos. Enquanto para $y$, seguindo a mesma abordagem, foram testados valores entre 1 e 20 sequencialmente e mais 5 valores entre 20 e 84 .

Depois de selecionados os valores, para cada par de parâmetros foi gerada uma floresta aleatória utilizando $C$ para o treinamento. Assim, foram obtidas 140 florestas aleatórias. Em seguida, as imagens contidas em $T$ foram submetidas a cada uma das 140 florestas para predição, com o intuito de investigar a acurácia alcançada.

Os dados obtidos no experimento foram tabulados, gerados gráficos e são apresentados e discutidos na próxima seção.

\section{Análise de Resultados}

A sintetização das acurácias obtidas em relação aos valores dos parâmetros $y$ e $B$ é mostrada na Tabela 1. Como pode ser observado, os resultados dos testes de predição apontam que a porcentagem de casos classificados corretamente entre todas as amostras testadas se manteve acima de $93 \%$ para $y \in[5,84]$ e $B \in[50,250]$. Para o restante, as florestas aleatórias apresentaram erros maiores que 7\%, uma taxa indesejável para o problema de reconhecimento de dígitos, conforme afirmado por Breiman (2001).

\begin{tabular}{|c|c|c|c|c|c|c|c|}
\hline $\begin{array}{c}\text { Númer0 } \\
\text { de } \\
\text { árvores/ } \\
\text { Valor de } \\
\boldsymbol{y}\end{array}$ & $\mathbf{5}$ & $\mathbf{1 0}$ & $\mathbf{5 0}$ & $\mathbf{1 0 0}$ & $\mathbf{1 5 0}$ & $\mathbf{2 0 0}$ & $\mathbf{2 5 0}$ \\
\hline $\mathbf{1}$ & 47,20 & 52,70 & $\mathbf{6 4 , 9 5}$ & 70,75 & 70,25 & 71,00 & 71,20 \\
\hline $\mathbf{2}$ & $\mathbf{6 3 , 9 5}$ & 74,65 & 86,20 & 88,75 & 88,50 & 88,60 & 89,25 \\
\hline $\mathbf{3}$ & $\mathbf{6 8 , 6 0}$ & $\mathbf{7 8 , 6 5}$ & 88,75 & 91,05 & 91,55 & 91,45 & 92,15 \\
\hline $\mathbf{4}$ & $\mathbf{7 4 , 9 5}$ & 82,40 & 91,30 & 92,15 & 92,55 & 92,90 & 93,20 \\
\hline $\mathbf{5}$ & $\mathbf{7 6 , 5 0}$ & 85,40 & 92,60 & 93,15 & 93,80 & 93,75 & 94,15 \\
\hline $\mathbf{6}$ & 79,25 & 84,90 & 93,00 & 94,10 & 94,20 & 94,35 & 94,45 \\
\hline $\mathbf{7}$ & 77,50 & 85,95 & 93,55 & 94,40 & 94,45 & 94,80 & 94,90 \\
\hline $\mathbf{8}$ & 79,45 & 87,55 & 93,60 & 93,70 & 94,45 & 94,50 & 94,95 \\
\hline $\mathbf{9}$ & 81,65 & 88,10 & 94,05 & 94,60 & 94,50 & 94,70 & 95,00 \\
\hline $\mathbf{1 0}$ & 82,30 & 88,05 & 94,15 & 94,50 & 94,85 & 94,90 & 94,95 \\
\hline $\mathbf{1 1}$ & 81,70 & 88,30 & 94,35 & 95,15 & 95,30 & 95,60 & 95,45 \\
\hline $\mathbf{1 2}$ & 81,85 & 88,60 & 94,90 & 95,35 & 95,45 & 95,60 & 95,75 \\
\hline $\mathbf{1 3}$ & 84,35 & 89,60 & 94,35 & 95,25 & 95,40 & 95,30 & 95,60 \\
\hline $\mathbf{1 4}$ & 85,65 & 89,15 & 94,60 & 95,20 & 95,25 & 95,40 & 95,85 \\
\hline $\mathbf{1 5}$ & 82,45 & 88,90 & 94,90 & 94,85 & 94,90 & 95,10 & 95,85 \\
\hline $\mathbf{1 6}$ & 83,70 & 88,80 & 94,85 & 95,95 & 95,60 & 95,75 & 95,85 \\
\hline $\mathbf{1 7}$ & 84,60 & 90,10 & 94,75 & 94,90 & 94,70 & 94,85 & 95,15 \\
\hline $\mathbf{1 8}$ & 85,90 & 90,95 & 94,85 & 95,50 & 95,60 & 96,75 & 96,90 \\
\hline $\mathbf{1 9}$ & 85,30 & 91,05 & 95,10 & 95,65 & 95,65 & 95,85 & 95,95 \\
\hline $\mathbf{2 0}$ & 84,35 & 90,70 & 94,90 & 95,85 & 95,95 & 95,75 & 94,95 \\
\hline $\mathbf{3 0}$ & 86,15 & 91,50 & 94,85 & 95,35 & 95,40 & 95,20 & 95,45 \\
\hline $\mathbf{4 0}$ & 87,25 & 92,50 & 95,05 & 96,00 & 96,10 & 96,30 & 96,45 \\
\hline $\mathbf{5 0}$ & 88,85 & 91,85 & 95,40 & 95,95 & 96,00 & 96,20 & 96,20 \\
\hline $\mathbf{6 0}$ & 87,30 & 92,75 & 94,95 & 95,60 & 95,80 & 96.15 & 93,30 \\
\hline $\mathbf{8 4}$ & 88,80 & 91,95 & 95,45 & 95,50 & 96,15 & 96,10 & 96,15 \\
\hline & & & & & & \\
\hline
\end{tabular}

Tabela 1 - Sintetização dos resultados em relação aos dois parâmetros estudados

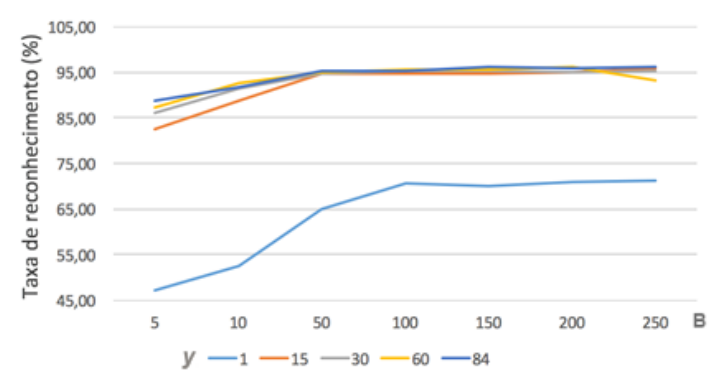

Figura 5. Reconhecimento caracteres $(y)$

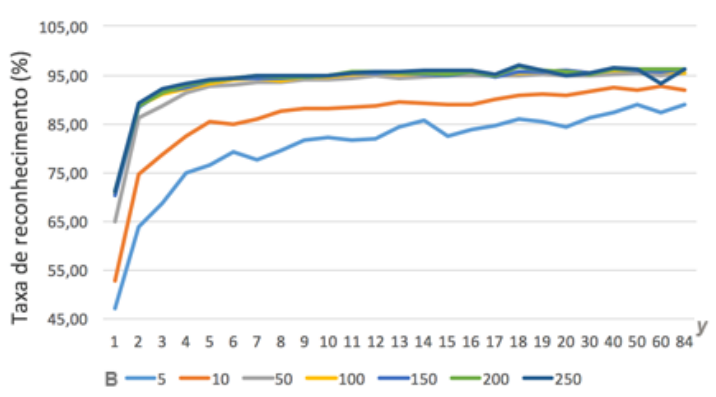

Figura 6. Reconhecimento caracteres $(B)$

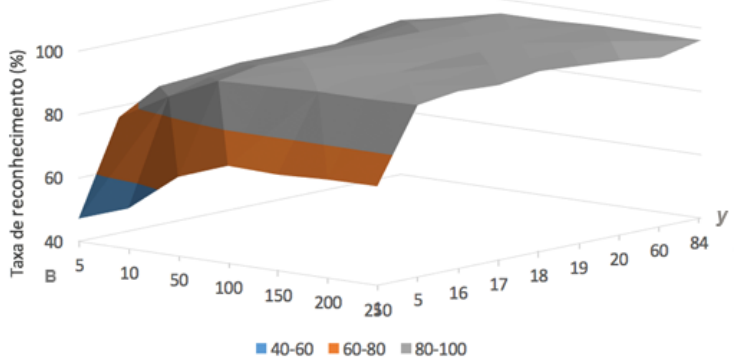

Figura 7. Diagrama das convergências

Analisando a influência do parâmetro $B$, observa-se uma tendência da taxa de reconhecimento se elevar para um número crescente de árvores. Entretanto, nota-se que 
o aumento verificado não é linear, o que permite entender que, a partir de 50 árvores, quando há um crescimento a precisão da floresta aleatória tende a sofrer variações menores. Esse comportamento pode ser observado Figura 5.

O gráfico da Figura 6 explicita os resultados com foco no número de recursos préselecionados aleatoriamente para o processo de divisão, o parâmetro $y$. Observa-se que, quando foi fixado o número de árvores $B$, as curvas da taxa de reconhecimento em relação ao número de características $y$, tem padrões variados. Para $1 \leq y \leq 6$ a taxa de reconhecimento vai aumentando, e permanece quase constante até $y=50$ e fica instável e menor até $y=84$. Nota-se também, que o mínimo é alcançado para $y=1$ quando o número de árvores é pequeno $B=5$.

A Figura 7 traz um diagrama, que representa as convergências em relação aos dois parâmetros analisados. Esse diagrama foi formado e mostra um sombreado cinza escuro para as maiores taxas de reconhecimento de caracteres em imagens em uma área definida em $y \in[6,50]$ e $B \in[100,250]$.

\section{Conclusão}

A descrição de florestas aleatórias feita por Breiman (2001) trouxe muitos benefícios para as diversas áreas que utilizam o aprendizado de máquina. O desenvolvimento dessas técnicas utilizando múltiplas árvores eficazes em altas dimensões possibilitou que diversos problemas de aprendizado de máquina tivessem sua dimensão ampliada substancialmente na última década, o que é resultado do interesse no estudo de textos e imagens, que muitas vezes necessitam de um grande número de variáveis.

A revisão bibliográfica corroborou para a compreensão dos desafios encontrados e possibilidades de exploração e validação teórica de alguns conceitos utilizados e analisados empiricamente. Além disso, ficou clara a importância dos métodos estudados para a solução de problemas nas mais diversas áreas atualmente.

O algoritmo de treinamento e classificação utilizando florestas aleatórias no âmbito deste estudo permitiu realizar testes preliminares e experimentos para verificar a acurácia das florestas aleatórias construídas. Analisando empiricamente os resultados dos experimentos, é possível afirmar que a acurácia obtida para valores de $y=18$ e $B=200$ é satisfatória para a classificação de dígitos numéricos escritos à mão presentes em imagens contidas em vídeos educacionais.

Em relação aos trabalhos analisados, que utilizaram apenas imagens contidas no MNIST, a presente pesquisa contribui com uma arquitetura de um algoritmo, composto pelos seguintes módulos: treinamento, pré-processamento e predição; que permite ao usuário escolher valores para os parâmetros necessários para o treinamento da floresta aleatória, o processamento de imagens geradas manualmente e com quaisquer resoluções, a entrada de uma imagem contendo vários dígitos, o reconhecimento e a classificação de um ou mais dígitos presentes em uma imagem.

Em síntese, esse algoritmo pode auxiliar na anotação semântica automática de vídeos educacionais que contenham dígitos numéricos. Entretanto, a expansão para a utilização de caracteres da tabela ASCII poderá ser explorada em futuras investigações. 
V Congresso Brasileiro de Informática na Educação (CBIE 2016)

Anais dos Workshops do V Congresso Brasileiro de Informática na Educação (CBIE 2016)

\section{Agradecimentos}

Os autores agradecem ao CNPq, Capes, Fapesp e MackPesquisa pelo auxílio financeiro.

\section{Referências}

Amit, Y., Blanchard, G. and Wilder, K. (2000). "Multiple randomized classifiers: MRCL".

Bernard, S., Adam, S. and Heutte, L. (2007) "Using random forests for handwritten digit recognition." Document Analysis and Recognition, 2007. ICDAR 2007. Ninth International Conference on. Vol. 2. IEEE.

Borges, P. R. S. (2015) "Incorporação e Recuperação de Metadados Semânticos em Vídeos Educacionais". Programa de Pós-Graduação em Engenharia Elétrica e Computação da Universidade Presbiteriana Mackenzie.

Breiman, L. (2001) "Random forests." Machine learning 45.1, 5-32.

de Castro, L. N. e Ferrari, D. G. (2016), "Introdução à Mineração de Dados: Conceitos Básicos, Algoritmos e Aplicações”, Saraiva.

Dietterich, T. (1998). "An experimental comparison of three methods for constructing ensembles of decision trees: Bagging, boosting and randomization". Machine Learning, 1-22.

Friedman, J., Hastie, T. and Tibshirani, R. (2001) "The elements of statistical learning." Vol. 1. Springer, Berlin: Springer series in statistics.

Ho, T. K. (1995) "Random decision forests." Document Analysis and Recognition. Proceedings of the Third International Conference on. Vol. 1. IEEE.

Ho, T. K. (1998) "The random subspace method for constructing decision forests." Pattern Analysis and Machine Intelligence, IEEE: 832-844.

Mitchell, T. M. (1997) "Machine learning. Burr Ridge", IL: McGraw Hill 45: 995.

Patti, Chanakya Reddy, et al. (2015) "Application of random forest classifier for automatic sleep spindle detection." Biomedical Circuits and Systems Conference (BioCAS), 2015 IEEE. IEEE.

$\mathrm{Pu}$, Xiaorong, et al. (2015) "Facial expression recognition from image sequences using twofold random forest classifier." Neurocomputing 168, 1173-1180.

Ristin, Marko, et al. (2016) "Incremental Learning of Random Forests for Large-Scale Image Classification." IEEE transactions on pattern analysis and machine intelligence 38.3 490-503.

Whelan, R., Garavan, H. (2013) "When optimism hurts: inflated predictions in psychiatric neuroimaging. " Biol. Psychiatry 75, 746-748.

Yao, Cong, et al. (2014) "Strokelets: A learned multi-scale representation for scene text recognition." Proceedings of the IEEE Conference on Computer Vision and Pattern Recognition. 